\title{
Vitamin-D Deficiency Predicts Infections in Young North Indian Children: A Secondary Data Analysis
}

\author{
Ranadip Chowdhury ${ }^{1}$, MD; Sunita Taneja ${ }^{1}$, PhD; Nita Bhandari ${ }^{1}$, PhD; Bireshwar Sinha ${ }^{1}$, MD; Ravi \\ Upadhyay ${ }^{1}$ MD; Maharaj Kishan Bhan ${ }^{2,3}$, MD; Tor A. Strand ${ }^{4,5, *}$, PhD \\ ${ }^{1}$ Centre for Health Research and Development, Society for Applied Studies, New Delhi 110016, India; \\ ranadip.chowdhury@sas.org.in; sunita.taneja@sas.org.in; nita.bhandari@sas.org.in; \\ bireshwar.sinha@sas.org.in; ravi.upadhyay@sas.org.in \\ ${ }^{2}$ National Science Professor, Indian Institute Technology, Delhi 110016, India; rajkbhan@gmail.com \\ ${ }^{3}$ Chair, Knowledge Integration and Translational Platform (KnIT), Biotechnology Industry Research \\ Assistance Council (BIRAC), New Delhi 110003, India \\ ${ }^{4}$ Department of Research, Innlandet Hospital Trust, 2381, Norway \\ ${ }^{5}$ Centre for International Health, University of Bergen, Bergen 5020, Norway \\ * Correspondence: tors@me.com \\ Trial Registration: www.cplinicaltrials.gov as NCT00717730 (Main Trial)
}

\begin{abstract}
Recent studies have demonstrated a relationship between poor vitamin D status and respiratory infections and diarrhea among young children. Acute lower respiratory infections (ALRI) and diarrhea are among the two most important causes of death in under- 5 children. In this analysis, we examine the extent to which vitamin-D deficiency $(<10 \mathrm{ng} / \mathrm{ml})$ predicts these outcomes using data from a randomized controlled trial (RCT) of daily folic acid and/ or vitamin B12 supplementation for six months in 6 to 30 months old children conducted in Delhi, India. Of the 960 subjects who had vitamin-D concentrations measured, 331(34.5\%) were vitamin-D deficient. We found, after controlling for relevant potential confounders (age, sex, breastfeeding status, wasting, stunting, underweight, anemia status at base line and season), the risk of ALRI was significantly higher among vitamin-D deficient (OR 1.26; 95\% CI: 1.03 to 1.55) compared to vitamin-D-replete children in the six months follow-up period. Vitamin-D status was not significantly associated with episodes of diarrhea and clinical pneumonia. The extent of causal relationship of vitamin-D status and ALRI needs to be explored in further studies.
\end{abstract}

Keywords: vitamin-D deficiency; ALRI; Indian children

\section{Introduction}

Vitamin D deficiency is considered to be the most common nutritional deficiency and often one of the undiagnosed medical conditions in the world [1]. The prevalence of vitamin D deficiency in young children is around $50-90 \%$ in the Indian subcontinent [2]. Vitamin D is primarily produced in the skin after exposure to ultraviolet radiation and less than $10 \%$ is derived from dietary sources [3].

Vitamin $\mathrm{D}$ is a potent immune-modulator of adaptive and innate immune responses [4]. In vitro studies have shown that 1,25-dihydroxyvitaminD3, the active metabolite of vitamin $\mathrm{D}$, is important for promoting and regulating immune responses [5,6]. Observational studies suggest a link between low vitamin D 
concentrations and an increased risk of lower and upper respiratory tract infections in infants and young children [7]. A recent prospective cohort study found that vitamin-D deficiency was associated with increased rates of diarrheal illnesses among school-aged children [8]. However, the extent to which vitamin D deficiency predicts these infections in young children is less clear.

The estimated incidence of pneumonia in children under 5 years is 0.29 episodes per child-year in developing countries, resulting in 151 million new episodes each year, of which $7-13 \%$ of cases are severe enough to be life-threatening and necessitate hospital admission [9]. In 2013, 25.3\% of deaths in children aged 1-59 months in India were due to pneumonia, totaling 150,169 deaths [10]. Globally, diarrhea causes $9 \%$ of under-5 deaths, most of these in developing countries [10]. Although a reduction has been observed in the incidence of diarrhea in resource-limited settings, the disease burden associated with recurrent enteric illnesses still remains a public health [11-12] problem that results in excess childhood mortality compared developed countries [13-15].

We conducted a randomized controlled trial (RCT) where children aged 6 to 30 months were supplemented daily with folic acid and/or vitamin B12 for six months. The main outcomes were the effect on the risk of acute lower respiratory infections (ALRI), clinical pneumonia and diarrhea. Enrolled participants were followed biweekly for respiratory and diarrheal morbidity [16]. Using data from this study we examined the extent to which vitamin-D deficiency $(<10 \mathrm{ng} / \mathrm{ml})$ at baseline predicted these outcomes during the 6 months follow-up period.

\section{Experimental Section}

\subsection{Subject}

The study was conducted in the low-to-middle socioeconomic settings of Tigri and Dakshinpuri in New Delhi with a total population of about 300,000 from January 2010 to February 2012. Details of the population have been described previously [16]. This randomized double-blind placebo-controlled trial with a factorial design enrolled 1000 children, and evaluated the impact of supplementation with folic acid, vitamin B12, or both on childhood infections [16]. The analyses in the current manuscript are restricted to the group of 960 children whose baseline vitamin-D levels were available.

\subsection{Definitions}

Diarrhea was defined as the passage of 3 or more loose or watery stools in a 24-h period. Two episodes of diarrhea were separated by a 72 hours or more diarrhea free period. ALRI was defined as cough or difficult breathing with an elevated respiratory rate above the age-specific cutoff values ( $\geq 50$ breaths $/ \mathrm{min}$ in infants and $\geq 40$ breaths/min in older children) according to WHO criteria, or cough or difficult breathing and lower chest in drawing [17]. Clinical pneumonia was defined either by a combination of cough with crepitations or bronchial breathing by auscultation or as an episode of ALRI associated with at least one of the following features: lower chest indrawing, convulsions, inability to drink or feed, extreme lethargy, restlessness or irritability, nasal flaring, or abnormal sleeping and difficulty in waking.

\subsection{Analytical Procedures}


Blood samples were obtained at baseline from all the children; $3 \mathrm{~mL}$ blood was collected in an evacuated tube containing EDTA (Becton Dickinson). The plasma was centrifuged at $\sim 450 \times \mathrm{g}$ at room temperature for $10 \mathrm{~min}$, separated, and transferred into storage vials and stored at $-20^{\circ} \mathrm{C}$ until analyzed. Plasma concentration of vitamin-D was measured by quantitative electro-chemiluminescence binding assay, with detection of 25(OH) $\mathrm{D}_{2}$, the hydroxylated forms of vitamin D2 (Roche Diagnostics, Mannheim , Germany) [18] at Christian Medical College, Vellore biochemistry laboratory.

\subsection{Ethics}

This study was conducted according the guidelines laid down in the Declaration of Helsinki and all procedures involving human subjects were approved by the Ethics committee at Society for Applied Studies, New Delhi, Christian Medical College Vellore and Norwegian Regional Committee for Medical and Health Research Ethics (REK VEST).

\subsection{Statistical Analysis}

Proportions and means (SD) or median (IQR) were calculated for categorical and continuous variables by Vitamin-D status at baseline. Vitamin D deficiency was defined at $<10 \mathrm{ng} / \mathrm{mL}(25 \mathrm{nmol} / \mathrm{L})$ [19]. The 6 months' follow-up period was divided into 26 periods of $7 \mathrm{~d}$ for every child. For a period to be included in the analyses, we required information on $4 \mathrm{~d}$ or more days of the given $7 \mathrm{~d}$ period. To account for interdependence of multiple observation periods in the same child, we used generalized estimating equations (GEE) with an autoregressive covariance-variance matrix taking time into account. In these models, occurrence of a new episode of diarrhea, ALRI, or clinical pneumonia in a child period was modeled as dependent variables and baseline vitamin $\mathrm{D}$ status as an independent variable. We included types of intervention received and other baseline variables as independent variables (age, sex, breastfeeding status, wasting, stunting, underweight, anemia status at base line and season) in the model to adjust for potential confounding. The model used a logit link, binomial variance, autoregressive correlation and robust standard error to yield odds ratio (OR). We used STATA version 14 (Stata Corporation, College Station, TX) for most statistical analyses. We used generalized additive models in the statistical software $\mathrm{R}$ version 3.1.2 (The R Foundation for Statistical Computing, Vienna, Austria) to explore nonlinear associations between the vitamin $\mathrm{D}$ level at baseline and ALRI incidence after adjustment for potential confounders [20]. We also used generalized additive model to explore nonlinear association between vitamin D level at baseline and season defined by period of enrollment in weeks. We considered an association to be statistically significant when the $P$ value was $<0.05$. Post-hoc calculations of statistical power showed that we had more than $90 \%$ power to detect at least $25 \%$ more episodes of ALRI during 6 months follow-up in the vitamin- D deficient group compared to vitamin-D non deficient group with the available sample, at $5 \%$ significance level.

\section{Results}


A total of 1000 children were included in the main trial. Blood samples for vitamin D were collected at baseline for 960 (96\%) children. Of these, 331 (34.5\%) children were Vitamin D deficient (<10 ng/ml). The baseline characteristics of the population by deficiency status are presented in Table 1. Approximately half of the enrolled children were boys and almost all (98\%) were ever breast fed. Over $36.4 \%$ of the children were stunted, $31 \%$ underweight, and $10.7 \%$ wasted. Approximately $70 \%$ of the children were anemic. Table 1: Baseline characteristics of Vitamin D deficient and non deficient children aged 6-30 months included in the analysis

$\begin{array}{ll}\text { Characteristics } & n=960\end{array}$

\section{Proportion of children}

Deficient $(<10 \mathrm{ng} / \mathrm{ml})$ Non deficient $(\geq 10 \mathrm{ng} / \mathrm{ml}$ )

Infant characteristics

Age at enrollment, months (mean, SD)

Proportion of children

$<12$ months

12 to 23 months

24 to 30 months

Boys

Ever breastfed

Prevalence of illness in previous 24 hours

Diarrhea

Cough or difficult breathing or fast

breathing

Anthropometric status

Mean(SD): Z score

Weight for Height Z score(WHZ)

$-0.86(0.92)$

$-1.56(1.24)$

Height for Age $Z$ score (HAZ)

Weight for Age Z score (WAZ)

Wasted (<-2 WHZ)

Stunted (<-2 HAZ)

35 (10.6)

117 (35.3)

$101(30.5)$

244 (73.7)

Anemia $(\mathrm{Hb}<11 \mathrm{~g} / \mathrm{dl})$

Socio-demographic characteristics

Mother's age, years (mean, SD)

$26.3(5.8)$

$331(34.5)$

$629(65.5)$

\section{Deficient}

$16.9(7.1)$

$91(27.5)$

$162(48.9)$

325 (98.2)

$17(5.1)$

$114(34.4)$

$-1.46(1.06)$

$8(5,10)$

$10(7,12)$

Father's schooling, years (median, IQR)
Non deficient

$n=629$

$15.8(7.0)$

$210(33.4)$

$306(48.7)$

$113(18.0)$

$328(52.2)$

$622(98.9)$

$32(5.1)$

$192(30.5)$

$-0.89(0.94)$

$-1.63(1.16)$

-1.52 (1.05)

68 (10.8)

$233(37.0)$

197 (31.3)

$424(67.4)$

25.6(4.1)

$7(0,10)$

$9(6,12)$

$72000(60000-144000)$

$84000(60000-138000)$ 
Figure 1 shows the relationship between vitamin-D according to weeks of enrolment. As distinct seasons are difficult to define in India, we divided the period of enrollments into weeks. The vitamin D concentrations were higher in the $24^{\text {th }}$ to $32^{\text {nd }}$ weeks which correspond to months (May- July) with more daylight. The baseline vitamin D levels were lower for children who were enrolled in the initial weeks of the year which correspond to months (January-February) and have less daylight.

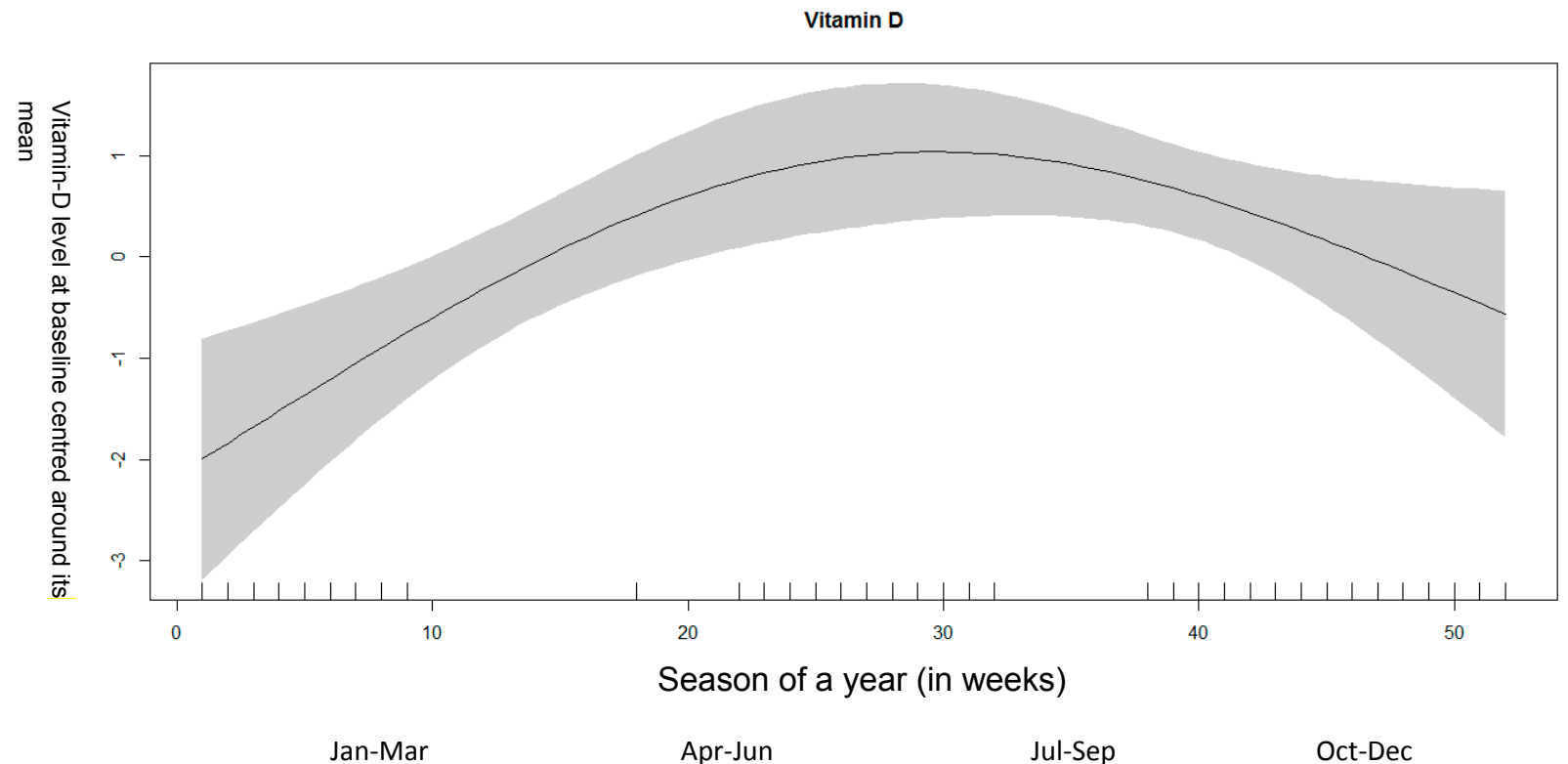

Figure 1: Associations between vitamin-D level at baseline and weeks of a year. The graph was constructed using generalized additive models in $\mathrm{R}$, the solid line depicts the association of vitamin-D level at baseline and season. The shaded area spans the $95 \%$ confidence interval of this association.

The diarrheal episodes in vitamin-D deficient and non-deficient children are shown in Table 2. There was no association between vitamin-D status and episodes of diarrhea overall and according to episodes of diarrhea lasting 6 days or more. The association between vitamin-D status and ALRI and clinical pneumonia are shown in Table 3. The incidence of ALRI was significantly higher among vitamin-D deficient children than in vitamin-D replete children (OR: 1.26; 95\% CI: 1.03-1.55). However, the incidence of clinical pneumonia was not significantly associated with vitamin D status (OR: 1.05; 95\% CI: 0.79-1.38).

Table 2: Incidence of diarrheal episodes in vitamin-D deficient and non deficient children 
Total child-years of follow-up

Episodes of diarrhea

Incidence density of diarrhea

per child year $(95 \% \mathrm{CI})$

Episodes of diarrhea lasting

$\begin{array}{lc}>=3 \mathrm{~d} & 328 \\ >=5 \mathrm{~d} & 182 \\ >=7 \mathrm{~d} & 114 \\ >=14 \mathrm{~d} & 34\end{array}$

Episodes of diarrhea with $>=6$

stools/on any day
162.3

775

4.78 (4.44 to 5.12$)$

328

182

114

34

200
308.5

1385

4.49 (4.26 to 4.73 )

1.07 (0.95 to 1.20$)$

568

339

218

63

409
1.07 (0.96 to 1.19$)$

0.98 (0.83 to 1.15$)$

0.95 (0.76 to 1.20$)$

1.01 (0.63 to 1.61 )

1.01 (0.83 to 1.22$)$

"Reference category: Non Deficient (Vitamin D level $\geq 10 \mathrm{ng} / \mathrm{ml}$ )

** ORs were calculated by using generalized estimating equations with a logit link, binomial variance, autoregressive correlation and robust standard error and adjusted for age, sex, breastfeeding status, wasted, stunted, underweight, anemia status at base line, season and type of interventions 
Table 3: Incidence of ALRI and clinical pneumonia in vitamin-D deficient and non deficient children

\begin{tabular}{|c|c|c|c|}
\hline & $\begin{array}{c}\text { Deficient } \\
\text { (Vitamin D level < } 10 \mathrm{ng} / \mathrm{ml})\end{array}$ & $\begin{array}{c}\text { Non Deficient } \\
\text { (Vitamin D level } \geq 10 \mathrm{ng} / \mathrm{ml} \text { ) }\end{array}$ & OR $(95 \% \mathrm{CI})^{* *}$ \\
\hline Total child-years of follow-up & 162.3 & 308.5 & \\
\hline Episodes of ALRI & 244 & 418 & \\
\hline $\begin{array}{l}\text { Incidence density of ALRI per } \\
\text { child year }(95 \% \mathrm{CI})\end{array}$ & $1.50(1.32$ to 1.70$)$ & 1.35 (1.23 to 1.49$)$ & $1.26(1.03$ to 1.55$)$ \\
\hline Episodes of clinical pneumonia & 144 & 294 & \\
\hline $\begin{array}{l}\text { Incidence density of clinical } \\
\text { pneumonia per child year }(95 \% \mathrm{CI})\end{array}$ & 0.89 (0.75 to 1.04$)$ & 0.95 (0.85 to 1.07$)$ & 1.05 (0.79 to 1.38$)$ \\
\hline
\end{tabular}

"Reference category: Non Deficient (Vitamin D level $\geq 10 \mathrm{ng} / \mathrm{ml}$ )

** ORs were calculated by using generalized estimating equations with a logit link, binomial variance, autoregressive correlation and robust standard error and adjusted for age, sex, breastfeeding status, wasted, stunted, underweight, anemia status at base line, season and type of interventions ALRI, acute lower respiratory infection

The association between baseline vitamin-D levels and incidence density of ALRI is depicted in Figure 2 . The ALRI incidence density increases with decreasing baseline vitamin-D concentrations. 


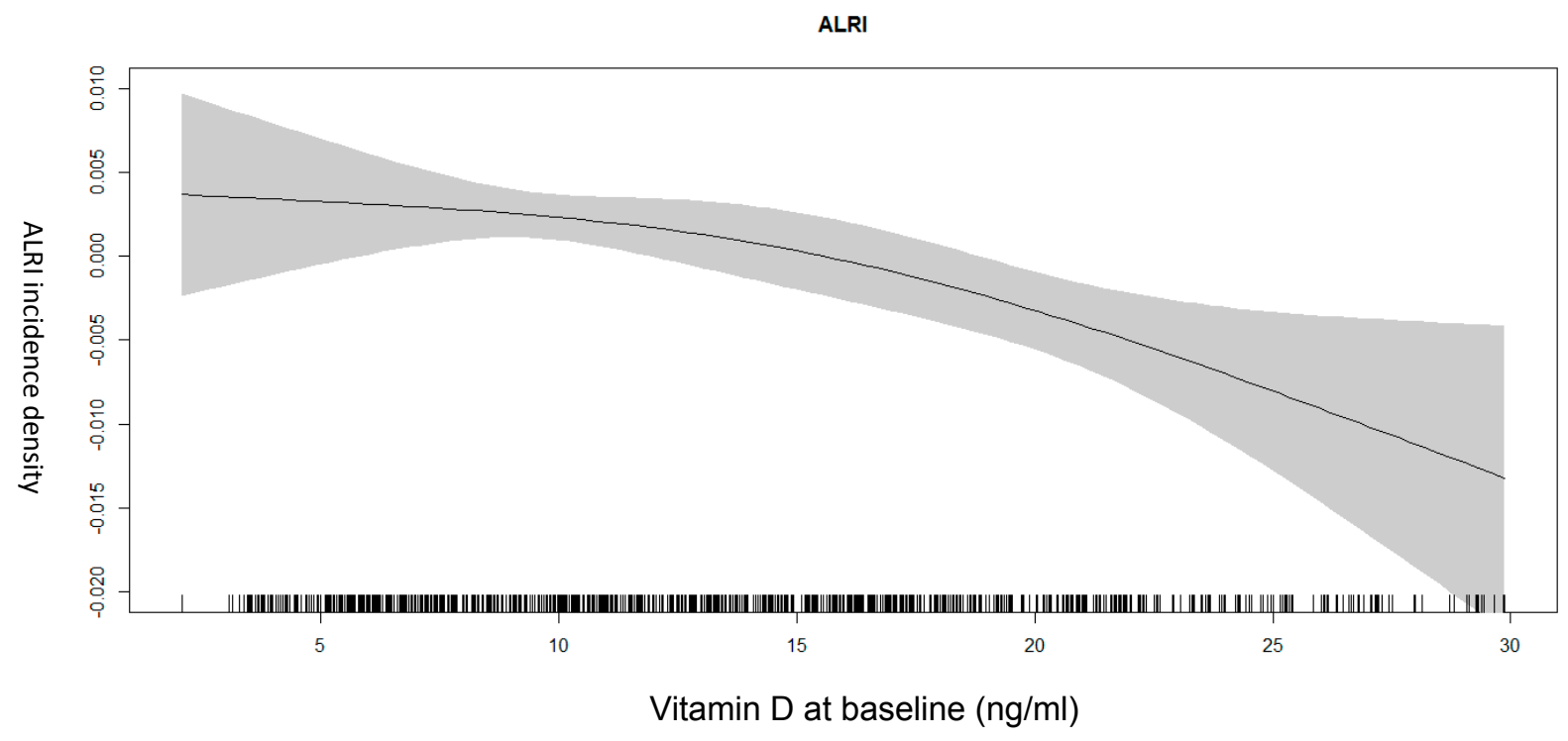

Figure 2: Associations between vitamin-D level at baseline and ALRI incidence density. The graph was constructed using generalized additive models in R, the solid line depicts the association of vitamin-D level at baseline and ALRI incidence density. The shaded area spans the $95 \%$ confidence interval of this association. 


\section{Discussion:}

We report the prevalence of vitamin D deficiency and its effect on the risk of common infections in young children. In the study population, we found a high prevalence of vitamin D deficiency which is consistent with other Indian studies [2]. However, a recent study from Nepal found that only $<5 \%$ of breastfed infants were vitamin-D deficient, even when a higher cut off $(<20 \mathrm{ng} / \mathrm{l})$ was used [21]. High prevalence of vitamin-D deficiency observed in our study setting, in spite of abundant sunlight may be because of an increased solar zenith angle, atmospheric pollution and type- $V$ skin types of the population [22]. More Ultra violet B (UVB) photons are absorbed by the stratospheric zone, and therefore very few of the UVB photons can penetrate to earth's surface to produce cutaneous pre-vitamin D3 with an increase in solar zenith angle (angular distance between an object in the sky, such as the sun, and an object directly overhead) especially in winter [23]. An earlier study also revealed high prevalence of vitamin D deficiency rickets in toddlers in Delhi (latitude: $28.35^{\circ} \mathrm{N}$ ) which has high atmospheric pollution [24]. A strong inverse correlation between vitamin-D status and latitude $(\mathrm{r}=-0.48 ; \mathrm{p}<0.0001)$ has been seen in various studies from India [23]. Skin pigmentation reduces UVB penetration into epidermis by $\sim 90 \%$ thereby limiting the production of previtamin D3 by the skin [23]. A recent study indicated that infants may get enough vitamin D from breast milk if their mothers take high-dose vitamin D supplements [25]. The complementary foods in the diets of Indian infants and children are primarily cereal based with less vitamin D [26]. This is also one of the contributing factors of vitamin-D deficiency among children in this setting where meteorological and environmental factors of vitamin-D deficiency already exist.

We found a significantly higher incidence density of ALRI among vitamin-D deficient children when compared to vitamin-D replete children. Similar findings have been shown in previous observational studies [27-29]. Vitamin-D acts as a link between TLR activation and antibacterial responses which in turns enhances production of cathelicidin (LL-37), an endogenous antimicrobial peptide which is highly expressed at natural barrier sites e.g. lungs [30].The protective role of vitamin-D against ALRI can be explained through its modulatory effect of both innate and adaptive immunity and regulatory function of inflammatory cascade [31-34].

We did not find any association with clinical pneumonia, a severe form of ALRI and vitamin D status. Observational studies have shown mixed results; while some studies showed evidence of association between vitamin-D status and clinical pneumonia, others did not find any association [35-38]. A recent systematic review concluded that there was no evidence of therapeutic vitamin D supplementation among under-5 children in the management of clinical pneumonia [39]. This result can be explained by that vitamin-D plays a role in the defense against infection but once infection has taken place, other factors determine its course and whether it will resolve or not. Vitamin D has distinct effects on the innate and adaptive immune responses and thus may explain different roles in pathogen-specific infection severity [40]. Given the complexity of interaction of vitamin-D with the immune system and inflammatory cascade, more research is needed to further define the specific role of vitamin $\mathrm{D}$ in enhancing immune function and reducing the severity of infections.

Poor vitamin D status was not associated with the incidence of diarrhea in our study although this has been shown in school age children [8]. Another randomized controlled trial with 3-monthly bolus supplementation with 100,000 IU of vitamin D3 among children aged 1 to 29 months, showed no effect on the risk of diarrheal illnesses [41]. The finding of this study underscores the need for further research to explore vitamin D serologic levels in relation to immune function and physiologic responses to gut infections. A recent study demonstrated that vitamin D receptor (VDR) polymorphisms play a role in inflammation, possibly resulting from alterations in gut permeability and microbial translocation, but whether this immune modulator function translates into reducing risk of diarrhea is unclear [42]. 
The strengths of our study are that the data are from a well conducted randomized controlled trial with very low attrition rates. We made multiple follow up visits for assessing ALRI, clinical pneumonia and diarrhea so that all episodes were well documented. Outcomes were clearly defined and assessed by highly trained field staff. Results were adjusted for several relevant confounders at baseline including nutritional status of children and the season of vitamin-D measurement.

We used an immunological method to measure vitamin-D concentration. Matrix effects are known to be a problem with immunoassays and can overestimate the vitamin D concentrations [43]. The lipophilic nature of 25OHD makes it particularly vulnerable to the presence of other lipids in the serum or plasma sample which changes the ability of the binding agent to associate with 25OHD in the sample and the standard in an equal method [44]. A further problem is that 25OHD cannot be accurately measured unless it is released from its specific binding protein.

The results of this study have important public health implications. As fortified foods have been recognized as an important source of vitamin D [45] such as fortification of oil, cereal powders and even salt, supplementation and fortification may help in preventing vitamin D deficiency and such public health interventions need serious consideration in the Indian context.

\section{Conclusion}

The present study demonstrates that vitamin D deficiency increases the risk of ALRI in children aged 6-30 months. Randomized controlled trials measuring the effect of supplementation of vitamin $\mathrm{D}$ in these setting should be prioritized. 


\section{Acknowledgements}

We acknowledge the input from Ratnasamy Selvakumar, Department of Biochemistry from Christian Medical College, Vellore, India for biochemical analysis. The Society for Applied Studies acknowledges the core support provided by the Department of Maternal, Newborn, Child and Adolescent Health, World Health Organisation, Geneva (WHO Collaborating Centre IND-096) and the Centre for Intervention Science in Maternal and Child Health (RCN Project No. 223269), Centre for International Health, University of Bergen (Norway). We also acknowledge the support extended by the Knowledge Integration and Technology Platform (KnIT), a Grand Challenges Initiative of the Department of Biotechnology and Biotechnology Industry Research Assistance Council (BIRAC) of Government of India and Bill \& Melinda Gates Foundation (USA).

\section{Authors' information and contributions}

RC, TS and ST conceptualized the study with contributions from all authors, and wrote the first draft. RC and TS did the statistical analysis with support from ST, NB, RU, BS and MKB. All authors contributed to the critical interpretation and writing of the paper and saw and approved the final version.

\section{Conflict of interest}

The authors declare no conflict of interest. The founding sponsors had no role in the design of the study; in the collection, analyses, or interpretation of data; in the writing of the manuscript, and in the decision to publish the results.

\section{References:}

1. Holick, M. F. Vitamin D: extraskeletal health. Rheumatic diseases clinics of North America 2012, 38, 141-60. doi: 10.1016/j.rdc.2012.03.013

2. Ritu, G.; Gupta, A. Vitamin-D Deficiency in India: Prevalence, Causalities and Interventions. Nutrients 2014,6,729-775. doi: 10.3390/nu6020729.

3. Norris, J.M. Can the sunshine vitamin shed light on type 1 diabetes. Lancet 2001, 358,14761478. DOI:10.1016/S0140-6736(01)06570-9

4. Kamen, D.L.; Tangpricha, V. Vitamin D and molecular actions on the immune system: modulation of innate and autoimmunity. J Mol Med. 2010,88,441-50. doi: 10.1007/s00109010-0590-9

5. Cantorna, M.T. Vitamin D and autoimmunity: is vitamin D status an environmental factor affecting autoimmune disease prevalence? Proc Soc Exp Biol Med. 2000,223,230-233.

PMID:10719834 
6. Pichler, J.; Gerstmayr, M.; Szeepfalusi, Z.; Urbanek, R.; Peterlik, M.; Willheim, M. 1-alpha, 25(OH)2D3 inhibits not only Th1 but also Th2 differentiation in human cord blood Tcells. Pediatr Res.2002,52,12-18. DOI:10.1203/00006450-200207000-00005

7. Esposito, S.; Lelii, M. Vitamin D and respiratory tract infections in childhood. BMC Infectious Diseases. 2015, 15,487. doi: 10.1186/s12879-015-1196-1.

8. Thornton, K.A.; Marín, C.; Mora, P.M.; Villamor, E. Vitamin D deficiency associated with increased incidence of gastrointestinal and ear infections in school-age children. Pediatr Infect Dis J. 2013,32,585-593. doi: 10.1097/INF.0b013e3182868989

9. Rudan, I.; Boschi, P.C.; Biloglav, Z.; Mulholland, K.; Campbell, H. Epidemiology and etiology of childhood pneumonia. Bull World Health Organ.2008,86,408-146. PMCID:PMC2647437

10. Liu, L.; Oza, S.; Hogan, D.; Perin, J.; Rudan, I.; Lawn, J.E. et al. Global, regional, and national causes of child mortality in 2000-13, with projections to inform post-2015 prorities: an updated systematic analysis. Lancet 2015,385,430-440. doi: 10.1016/S0140-6736(14)61698-6

11. Fischer, C.L.; Perin, J.; Aryee, M.J.; Boschi, P.C.; Black, R.E. Diarrhea incidence in low- and middle-income countries in 1990 and 2010: a systematic review. BMC Public Health. 2012,12,220. doi: 10.1186/1471-2458-12-220

12. Bhutta, Z.A.; Das, J.K. Global burden of childhood diarrhea and pneumonia: what can and should be done? Pediatrics. 2013,131,634-636. doi: 10.1542/peds.2012-3737

13. Guerrant, R.L.; Oriá, R.B.; Moore, S.R.; Oriá, M.O.; Lima, A.A. Malnutrition as an enteric infectious disease with long-term effects on child development. Nutr Rev. 2008,66, 487-505. doi: 10.1111/j.1753-4887.2008.00082.x.

14. Rodríguez, L.; Cervantes, E.; Ortiz, R. Malnutrition and gastrointestinal and respiratory infections in children: a public health problem. Int J Environ Res Public Health. 2011,8,11741205. doi: 10.3390/ijerph8041174

15. Checkley, W.; Buckley, G.; Gilman, R.H. Childhood Malnutrition and Infection Network. Multi-country analysis of the effects of diarrhoea on childhood stunting. Int J Epidemiol. 2008,37,816-830. doi: 10.1093/ije/dyn099

16. Taneja, S.; Strand, T.A.; Kumar, T.; Mahesh, M.; Mohan, S.; Manger, M.S. Folic acid and vitamin B-12 supplementation and common infections in 6-30-mo-old children in India: a randomized placebo-controlled trial. Am J Clin Nutr. 2013,98,731-737. doi: 10.3945/ajcn.113.059592

17. World Health Organization. Integrated management of childhood illness. Geneva, Switzerland: WHO, 2003.

18. Cobas E411 Vitamin D Total Reagent Insert (06268668001V1). Roche Diagnostics. URL: http://www.captodayonline.com/productguides/instruments/automated-immuno assayanalyzers-july-2012/roche-diagnostics-cobas-e411-immunoassay-analyzers-june-2011.html (accessed on 13/09/2016)

19. Spiro, A.; Buttriss, J.L. Vitamin D: An overview of vitamin D status and intake in Europe. Nutrition Bulletin 2014,39,322-350. DOI:10.1111/nbu.12108

20. Wood, S.N. Modelling and smoothing parameter estimation with multiple quadratic penalties. JR Stat Soc B 2000,62, 413-428. Stable URL: http://www.jstor.org/stable/3088868

21. Ulak, M.; Chandyo, R.K.; Thorne L.A.L.; Henjum, S.; Ueland, P.M.; Midttun, O. Vitamin Status among Breastfed Infants in Bhaktapur, Nepal. Nutrients. 2016; 8. doi: 10.3390/nu8030149

22. Harinarayan, C. V.; Joshi, S. R. Vitamin D status in India--its implications and remedial measures. The Journal of the Association of Physicians of India 2009, 57, 40-48. PMID:19753759

23. Harinarayan, C. V.; Holick, M. F.; Prasad, U. V.; Vani, P. S.; Himabindu, G.,Vitamin D status and sun exposure in India. Dermato-endocrinology 2013, 5 (1), 130-141. doi: 10.4161/derm.23873

24. Agarwal, K.S.;Mughal, M.Z.; Upadhyay, P.; Berry, J.L.; Mawer, E.B.; Puliyel, J.M. The impact of atmospheric pollution on vitamin D status of infants and toddlers in Delhi, India. Arch. Dis. Child. 2002,87,11-13. PMCID: PMC1719192 
25. Hollis, B.W.; Wagner, C.L.; Howard, C.R.; Ebeling, M.; Shary, J.R.; Smith, P.G. Maternal Versus Infant Vitamin D Supplementation During Lactation: A Randomized Controlled Trial. Pediatrics. 2015,136, 625-634. doi: 10.1542/peds.2015-1669.

26. National Institute of Nutrition, India. Food composition tables. In Nutritive Value of Indian Foods, eds C Gopalan, BVR Sastri, SC Balasubramanian. Hyderabad: National Institute of Nutrition, Indian Council of Medical Research. 1996,45-95

27. Wayse, V.; Yousafzai, A.; Mogale, K.; Filteau, S. Association of subclinical vitamin D deficiency with severe acute lower respiratory infection in Indian children under $5 \mathrm{y}$. Eur J Clin Nutr 2004,58,563- 567. DOI:10.1038/sj.ejcn.1601845

28. Karatekin,G.; Kaya, A.; Salihoglu, O.; Balci, H.; Nuho, A. Association of subclinical vitamin $\mathrm{D}$ deficiency in newborns with acute lower respiratory infection and their mothers. European Journal of Clinical Nutrition.2009,63, 473-477. DOI:10.1038/sj.ejen.1602960

29. Roth, D.E.; Shah, R.; Black, R.E.; Baqui, A.H. Vitamin D status and acute lower respiratory infection in early childhood in Sylhet, Bangladesh. Acta Paediatrica 2010,99, 389-393. doi: 10.1111/j.1651-2227.2009.01594.x.

30. Quraishi, S. A.; Bittner, E. A.; Christopher, K. B.; Camargo, C. A., Jr. Vitamin D status and community-acquired pneumonia: results from the third National Health and Nutrition Examination Survey. PloS one 2013, 8, e81120.

31. Sun, J. Vitamin D and mucosal immune function. Curr Opin Gastroenterol. 2010,26,591-595. doi: 10.1097/MOG.0b013e32833d4b9f.

32. Esposito, S.; Lelii, M. Vitamin D and respiratory tract infections in childhood. BMC Infectious Diseases 2015,15, 487. doi: 10.1186/s12879-015-1196-1.

33. Liu, P.T.; Stenger, S.; Li, H.; Wenzel, L.; Tan, B.H.; Krutzik, S.R. Toll-like receptor triggering of a vitamin D - mediated human antimicrobial response. Science. 2006,311,1770-1773. DOI:10.1126/science.1123933

34. Bikle, D.D. Vitamin D and the immune system: role in protection against bacterial infection. Curr Opin Nephrol Hypertens. 2008,17,348-352. doi: 10.1097/MNH.0b013e3282ff64a3.

35. Muhe L, Lulseged S, Mason KE, Simoes EA. Case-control study of the role of nutritional rickets in the risk of developing pneumonia in Ethiopian children. Lancet. 1997;349:1801-4.

36. Ren J, Sun B, Miao P, Feng X. Correlation between serum vitamin D level and severity of community acquired pneumonia in young children. Zhongguo Dang Dai Er Ke Za Zhi. 2013;15:519-21.

37. Roth,D.; Jones, A.; Prosser, C.; Robinson, J.; Vohra, S. Vitamin D status is not associated with the risk of hospitalization for acute bronchiolitis in early childhood. Eur J Clin Nutr 2009,63,297-309

38. Sakka, A.S.E.; Iman, S.S.; Amer, H.A.; Moustafa, S.A. Vitamin D deficiency and low hemoglobin level as risk factors for severity of acute lower respiratory tract infections in Egyptian children: A case-control study. Egyptian Pediatric Association Gazette. 2014, 62,1-7 doi: http://dx.doi.org/10.1016/j.epag.2013.12.001

39. Das, R. R.; Singh, M.; Panigrahi, I.; Naik, S. S, Vitamin d supplementation for the treatment of acute childhood pneumonia: a systematic review. ISRN pediatrics 2013, 2013, 459160. doi: $10.1155 / 2013 / 459160$.

40. Wei, R.; Christakos, S. Mechanisms underlying the regulation of innate and adaptive immunity by vitamin D. Nutrients 2015,7, 8251-8260. doi: 10.3390/nu7105392

41. Aluisio, A.R.; Maroof, Z.; Chandramohan, D.; Bruce, J.; Mughal, M.Z.; Bhutta, Z. Vitamin $\mathrm{D}(3)$ supplementation and childhood diarrhea: a randomized controlled trial. Pediatrics. 2013,132,e832-840. doi: 10.1542/peds.2012-3986.

42. Al-Daghri, N.M.; Guerini, F.R.; Al-Attas, O.S.; Alokail, M.S.; Alkharfy, K.M.; Draz, H.M. Vitamin $\mathrm{D}$ receptor gene polymorphisms are associated with obesity and inflammosome activity. Plos One. 2014,9,e102141. doi: 10.1371/journal.pone.0102141

43. Costelloe, S.J.; Woolman, E.; Rainbow, S.; Stratiotis, L.O.G.G.; Whiting, S. Is highthroughput measurement of 25-hydroxyvitamin D3 without 25- hydroxyvitamin D2 
appropriate for routine clinical use? Anal Clin Biochem 2009,46,86-87. doi: 10.1258/acb.2008.008157.

44. Hollis, B.W. Editorial: The determination of circulating 25-hydroxyvitamin D: no easy task. J Endocrinol Metab 2004,89,3149-3151. DOI:10.1210/jc.2004-0682

45. Lamberg-Allardt, C. Vitamin D in foods and as supplements. Prog Biophys Mol Biol. 2006,92,33-38.

(C) 2016 by the authors; licensee Preprints, Basel, Switzerland. This article is an open access article distributed under the terms and conditions of the Creative Commons by Attribution (CC-BY) license (http://creativecommons.org/licenses/by/4.0/). 